\title{
Comparative Analysis of Mechanical Properties of Common Carotid Artery between Young and Old Specimen \\ Z Qin ${ }^{1}, \mathrm{G} \mathrm{Li}^{2}, \mathrm{~N} \mathrm{Liu}^{1}, \mathrm{C}$ Zhao ${ }^{1}, \mathrm{H} \mathrm{Zhao}^{1}$
}

\begin{abstract}
Objective: This study aimed to perform the carotid artery stretching experiments with young and elderly corpse for the study of carotid atherosclerosis pathogenesis and biomechanical basis.

Methods: 15 carotid arteries were randomly selected from young and elderly groups, respectively, for the longitudinal tensile test, and 1 specimens of each group was randomly selected for the morphological observation.
\end{abstract}

Results: The maximum longitudinal tensile stress, maximum strain, elastic limit strain of middle cerebral artery in young group were greater than those of elderly group $(\mathrm{P}<0.05)$, while the elastic modulus of middle cerebral artery in young group was less than that of elderly group $(\mathrm{P}<0.05)$.

SEM results showed that the muscle cells of middle cerebral artery in young group were in neat arrangement, with intact elastic membrane, while the muscle cells of elderly carotid artery showed active hyperplasia, some elastic membrane were ruptured, with disorganized arrangement.

Conclusions: The morphology and longitudinal tensile properties of carotid artery in elderly group of changed.

Keywords: Carotid artery, elderly, longitudinal stretching mechanical properties, youth

From: ${ }^{1}$ Department of Neurosurgery, Sino-Japanese Friendship Hospital of Hilin University, Jilin 130022, China, ${ }^{2}$ Department of Otolaryngology Head and Neck Surgery, Sino-Japanese Friendship Hospital of Jilin University, Jilin 130021, China.

Correspondence: Dr H Zhao, Department of Neurosurgery, Sino-Japanese Friendship Hospital of Jilin University, Jilin 130022, China. E-mail: zhongjunzhangcn@126.com

West Indian Med J

DOI: $10.7727 /$ wimj.2015.218 


\section{INTRODUCTION}

Carotid artery is the predilection site for carotid atherosclerosis, which is the most frequently involved in systemic atherosclerosis. Carotid atherosclerosis and stenosis are extremely easy to cause the cardiovascular and cerebrovascular diseases. Domestic and foreign scholars have performed on a lot of researches about the pathogenesis of atherosclerosis, stroke prevention, diagnosis and treatment (1-12). Homburg et al. (13) have carried out the correlation analysis on the relationship of CA and cerebral infarction, they believe that CA can be used as one of the most important risk indicator of cerebral infarction prediction; Simova et al. (14) have studied the relationship of media layer-thickness of carotid intima and CA and find that, the intima membrane thickness can be seen as the marker of atherosclerotic changes. Parmar et al. (15) have studied the relationship between stroke onset with the unstable carotid artery plaques or "vulnerable plaques" of ischemic stroke, and the results show that the complex carotid artery plaque was closely related to ischemic stroke. Sadekova et al. (16) have studied the effect of carotid artery calcification on cerebral arterial stiffness in mice by using $\mathrm{CaCl}_{2}$ deposition and find that, the carotid artery calcification can induce the cerebral superoxide anion generation and increased neurodegenerative diseases, with decreased carotid artery elasticity, compliance and distensibility. The mouse model of $\mathrm{CaCl}_{2}$ deposition in carotid artery conforms with the feature and specificity of arterial stiffness, and can be used to study the effects of arterial stiffness on the brain. Khorvash et al. (17) have comparatively analyzed the relationship between carotid artery intima-media thickness and type D personality in 
40-60 years old persons, and measured the carotid artery intima-media thickness in placebo group and type D personality group using Doppler ultrasound. Results show that, the average carotid artery intima-media thickness between two groups is no significantly different $(P=0.19)$. However, there are $22.9 \%$ and $48.6 \%$ of individuals with abnormal intima-media thickness in placebo group and type D personality group, with significant difference between them $(P=0.001)$. Type D personality can increase the incidence of cardiovascular disease, especially for stroke and myocardial infarction due to hormonal imbalance, which lead to artery vasospasm and atherosclerosis. Therefore, it is necessary to conduct evaluation and treatment for patients with increased risk of atherosclerosis and myocardial infarction. The elderly metabolic syndrome (MS), such as hypertension, hyperglycemia, dyslipidemia and obesity, was the frequently-occurring and common diseases towards the elderly, and the independent risk factors of cardiovascular diseases. Von Hafe et al. (18) reported that the prevalence rate of elderly MS was $43.5 \%$. The morbidity and mortality of elderly heart and cerebrovascular diseases were even higher. The main pathological basis of heart and cerebrovascular diseases were the atherosclerosis. In the recent years, the carotid had become a "window" that could reflect the systemic atherosclerotic lesions.

Jackson et al. (19) have copied rabbit carotid artery injury model and decreased the carotid artery tension with heteropleural transplantation method. Result show that, the reduced longitudinal tension can lead to vascular tissue reconstruction and arterial 
distortion. The size of the longitudinal tension has an important influence on the arterial injury transplantation.

There are rare studies of longitudinal tensile with the fresh cadaver carotid artery of Chinese people and fresh elderly carotid artery. It is deduced that the elderly carotid artery will change due to the changes of mechanical properties of CA tissue morphological changes. There are some differences between the mechanical properties of young and elderly carotid artery, which must verify through the tests. In view of this, the author performed the longitudinal tensile test on normal fresh carotid artery and elderly carotid artery of people cadaver, to comparatively analyze the tensile mechanical properties of fresh and elderly carotid artery of Chinese people cadaver, discuss the pathogenesis of atherosclerosis and cerebral infarction from the biomechanical perspectives.

\section{SUBJECTS AND METHODS}

\section{Subjects}

The young carotid arteries were obtained from 10 fresh normal adult male corpses, aged 22-25 years old, and the senile carotid arteries were from 10 elderly male bodies, aged 65-70 years old, who had the history of atherosclerosis and high blood pressure before the death. The bodies were provided by College of Basic Medical Science, Jilin University. The body was dissected within $24 \mathrm{hr}$ of death: the body was lied on the back, with a wooden pillow padded under the rear part of neck, so that the head could be lied back as far as possible. The jugular vein incision was made from the middle 
chin, along the midline and to the sternum, along the upper end of incision and the lower jaw, the incision was expanded to the bilateral mastoid bones, along the lower end of incision and the lateral side of clavicle, the incision was expanded to the acromion. Then along the anterior neck midline, close to the surface of platysma muscle, the skin was dissected and turned back to the front edge of trapezius muscle, thus the platysma muscle was revealed. The platysma muscle was then cut along the edge of clavicle, followed by flipped up close to the deep side until the basis mandibulae, the cervical plexus branches of sternocleidomastoid surface could then be recognized; found the anterior jugular vein from the superficial fascia on the bilateral sides of anterior neck midline, and found the initial segment of external jugular vein from the posterior mandibular angle, thus the external jugular vein could be seen obliquely pass through the sternocleidomastoid surface and penetrate the deep fascia, then removed all the superficial fascia. Cleaned and observed the cuff fascia, and it could be seen that it wrapped the sternocleidomastoid and trapezius, and linked up to the hyoid body; then cut the cuff fascia along the upper edges of sternum and clavicle, flipped up, and stripped the cuff fascia on the surface of sternocleidomastoid. The heads of sternum and clavicle of sternocleidomastoid were cut off, separated and turned upwards this muscle to observe the deep carotid sheath, under which surface the hypoglossal nerve loop could be seen, with deep lateral cervical lymph nodes surrounded, and divided into two groups (up and down) from the scapular hyoid muscle. The carotid sheath was then vertically incised to reveal the common carotid artery, according to experimental needs, 10 left common carotid arteries and 10 right 
common carotid arteries were obtained. The specimens were placed in the physiological saline for the future use. And the use of specimens was approved by the ethics committee of Medicine Department of Jilin University.

\section{Preparation of the carotid artery specimens}

20 young and elderly carotid artery specimens, respectively, were stored for one day, then removed the carotid artery. The S-5 sterile plastic handle scalpel, Lianheyikang, Huai'an, Jiangsu Province, was used to cut and obtain $2015 \mathrm{~mm}$-long samples in the proximal site of the elderly common carotid artery group, as well as 20 samples from the same site of the youth group for the tensile test. And in both elderly and young groups, a $5 \mathrm{~mm}$ length sample from the bifurcation of the common carotid artery was used for the tissue morphological observation.

\section{Tensile test method}

The laboratory equipment was Css4410 automatic-controlling electronic universal testing machine (Changchun, Jilin, China), Changchun Institute of Testing Machine, with ambient temperature box. The reading microscope, the $3^{\text {rd }}$ Optical Instrument Factory (Changchun, Jilin, China), was used to measure the lengths and diameters of specimens in each group. The common carotid artery sample was $15 \mathrm{~mm}$ in length and $1.19-1.21 \mathrm{~mm}$ in thickness, with the inner diameter as $6.5-6.8 \mathrm{~mm}$ outer diameter as 8.89-9.12 mm. Firstly, each sample was performed pre-emptive adjustment according to the reference method (20-22). The experiment simulated the normal 
body temperature at $(36.5 \pm 1.0){ }^{\circ} \mathrm{C}$ conditions, the carotid artery specimens of young and elderly groups were clamped in the test machine, respectively, then applied the elongation load at the speed of $0.5 \mathrm{~mm} / \mathrm{min}$, at the same time, continuously leached some solution to the sample to maintain the humidity of the sample during the test. After the experiment, the computer would automatically output the stress, strain, elastic modulus under $10.7 \mathrm{kpa}(80 \mathrm{mmHg}), 16.0 \mathrm{kpa}(120 \mathrm{mmHg})$ and $225 \mathrm{kpa}(170$ $\mathrm{mmHg}$ ), and the stress - strain curve.

\section{SEM observation of the ultrastructure of carotid arterial cross section}

SEM was performed to observe the ultrastructure of the carotid arterial cross section in young and elderly groups: randomly selected 1 sample from the young and elderly groups, respectively, cut into pieces of $5 \mathrm{~mm}$, pre-fixed with $4 \%$ glutaraldehyde and post- fixed with $1 \%$ osmium tetroxide, then dehydrated in acetone and dried at the critical point, coated with gold film under vacuum, then the field emission SEM (Carl Zeiss Company, Jena, Germany) was performed to observe the morphology of carotid artery wall.

\section{Statistical analysis}

Data were expressed as mean \pm SD. Statistical analysis was performed using SPSS 11.0 statistical software. Single-factor randomized group design was used for analysis, and Dunnett's T3 test was applied to all pair-wise comparisons. $P<0.05$ was considered as statistically significant. 


\section{RESULTS}

\section{Sample number analysis}

The total sample number of carotid artery tensile test in the young and elderly groups was 40, all included in the results analysis, without any loss value. The 2 carotid artery tissue morphology samples of young and elderly groups were all included in the final analysis.

\section{Longitudinal tensile results of carotid artery specimens}

The experimental data of longitudinal tensile test were shown in Table 1, the data of the two groups were curve fitted with a computer, and the stress - strain curves were shown in Figure 1.

The tensile test results showed that the maximum stress of the elderly samples was $227.2 \pm 7.3 \mathrm{kpa}$, maximum strain was $67.7 \pm 5.0 \%$, and the elastic limit strain was $29.3 \pm 2.6 \%$, the elastic modulus at $13.3 \mathrm{kpa}$ stress was $98.5 \pm 1.8 \mathrm{kPa}, 102.5 \pm$ $2.3 \mathrm{kPa}$ at $16.0 \mathrm{kpa}$ stress and $106.1 \pm 1.5 \mathrm{kPa}$ at $22.5 \mathrm{kpa}$ stress, while towards the young specimens, the maximum longitudinal tensile stress was $254.8 \pm 13.1 \mathrm{kPa}$, the maximum strain was $90.6 \pm 10.6 \%$, the elastic limit strain was $39.5 \pm 2.8 \%$, the elastic modulus was $93.9 \pm 5.6 \mathrm{kPa}$ at $13.3 \mathrm{kpa}$ stress, $96.3 \pm 1.4 \mathrm{kPa}$ at $16.0 \mathrm{kpa}$ stress and $98.4 \pm 1.1 \mathrm{kPa}$ at $22.5 \mathrm{kpa}$ stress. The maximum stress, maximum strain and elastic limit strain of young group were larger than those of the elderly groups $(P<0.05)$, while the elastic modulus at $13.3 \mathrm{kpa}, 16.0 \mathrm{kpa}$ and $22.5 \mathrm{kpa}$ stress were less than the elderly group $(P<0.05)$. 
In Figure 1, the stress was the ordinate, and the strain was the abscissa. It could be concluded that when the stress was $16.0 \mathrm{kpa}$, the two stress - strain curves exhibited exponential relationship, the stress and strain of young group were greater than those of elderly group. When the stress exceeded $16.0 \mathrm{Kpa}$, the strain of both young and elderly groups increased rapidly, along with the increasing stress-strain curve, slope increased, the stress-strain relationship exhibited disproportionate change, the continuous force loading would result in the increaseing deformation of the vascular wall until the sample was damaged.

\section{Establishment of stress-strain functional relations}

From the measured data, the dependent relations and the total deviation among variables were investigated for the creation of the stress-strain functional relations:

$$
\begin{aligned}
& \text { Young group: } \sigma(\varepsilon)=0.0000 \mathrm{e}^{5}-0.0051 \mathrm{e}^{4}+0.8803 \mathrm{e}^{3}+2.6361 \mathrm{e}^{2} \\
& \text { Elderly group: } \sigma(\varepsilon)=-0.0000 \mathrm{e}^{5}+0.0054 \mathrm{e}^{4}+0.7930 \mathrm{e}^{3}+5.1093 \mathrm{e}^{2}
\end{aligned}
$$

\section{Histological analysis of longitudinal section on young and elderly groups}

In order to analyze the tissue morphology of longitudinal section in both young and elderly groups, SEM was performed to observe the morphological changes of longitudinal sections. The longitudinal optical micrographs of carotid artery in young and elderly groups were shown in Figures 2 and 3, respectively.

The morphology observation results showed that the carotid arterial intimal thickening of elderly group was apparent, the proliferation of a large number of 
smooth muscle cells (SMC) could be seen under the intima, with visible lipid plaque and the accumulation of foam cells, the internal elastic lamina and the elastic lamina degenerated, fractured and disintegrated, a lot of plaques and inflammatory cell infiltration could be seen in intima and tunica media, the tissue morphology exhibited the characteristics of atherosclerosis (Figure 3). While the structures of carotid artery wall layers in young group were normal, without atherosclerotic plaque, the intimal was smooth, and the elastic fibers in the tunica media were normal (Figure 2).

\section{DISCUSSION}

In this study, the experimental methods towards both young and elderly corpse carotid artery specimens, namely sampling, sample preservation, sample pre-tune treatment, experimental ambient temperature, experimental speed, and sample interventions for tissue morphological observation specimens, were all consistent, so the experimental results were reliable. Results of this study show that, there are obvious morphology and mechanical property changes of carotid artery in elderly group due to atherosclerosis, with significant difference from young group, which is consistent with expectation.

This study found that, when the stress did not exceed the normal diastolic pressure $(16.0 \mathrm{kpa})$, the carotid arterial strain increased rapidly in both two groups, when the stress exceeded $16.0 \mathrm{kpa}$, the strain rose slowly. At the beginning, the strain exhibited fast change speed, indicating the inherent expansion pressure inside the common carotid artery was less than the partial pressure, leading to faster 
water-spilling-out speed, and with continuous fluid outflow from the carotid artery, the difference between swelling pressure and partial pressure decreased, so the changes slowed down in the later stage. The feature, that the deformation of carotid artery wall under the outer load changed slowly instead of rapidly with the increasing of stress, could play a protective role when in emergency, there was no unusual, nor any clinical symptoms in the cerebral artery while the blood pressure increased. The morphology observation of both young and elderly groups showed that in the elderly group, carotid arterial intimal thickening was apparent, the proliferation of a large number of SMC could be seen in the intima, with visible plaques and the accumulation of foam cells, the internal elastic lamina and the elastic lamina degenerated, fractured and disintegrated, a lot of plaques and inflammatory cell infiltration could be seen in intima and tunica media, the tissue morphology exhibited the characteristics of atherosclerosis. While the structures of carotid artery wall layers in young group were normal, without atherosclerotic plaque, the intimal was smooth, and the elastic fibers in the tunica media were normal. The analysis believed that the carotid artery of the elderly group would appear atherosclerosis and proliferation of a large number of SMC under the arterial intima might because of the aging period of elderly body, the internal elastic lamina and the elastic lamina degenerated, fractured and disintegrated, leading to the changes of content and orientating direction of the elastic fibers and collagen fibers, therefore the flexibility and toughness decreased, while the hardness increased, namely the elastic modulus was improved. Long-term high blood pressure and high stress-induced carotid artery remodeling in elderly 
people led to the changes of blood vessels functions, therefore the arterial compliance decreased, and the mechanical properties of the vascular wall changed. CA has a very close relationship with cerebral infarction, and is the risk factor for cerebral infarction. After the formation of carotid artery plaques, the stress of the arterial wall would increase and the impact of the unstable blood would rupture plaque in hypertensive state, then activate the platelets, started the fibrinolytic system, which would gradually develop into cerebral infarction. The experimental results supported the view that the mechanical property changes of the vascular wall were one of the pathogenesis of atherosclerosis and cerebral infarction.

In this study, the maximum strain of carotid artery in human cadaver was $90.6 \pm$ $10.6 \%$, less than the pig carotid maximum tensile strain by Clerin (17), indicating that there were differences in tensile mechanical properties of the common carotid artery among different species. In the past, the mechanical properties of the common carotid artery studies mainly used the animal corpses carotid artery, and mostly focused on 1D longitudinal stress - strain relations, with the speed of longitudinal tensile experiments was faster than $0.5 \mathrm{~mm} / \mathrm{mim}$. In this study, the difference from previous studies was that the test speed was $0.5 \mathrm{~mm} / \mathrm{mim}$, with this speed, the maximum stress, strain data, the elastic modulus data at $13.3 \mathrm{kpa}, 16.0 \mathrm{kpa}$ and $17.8 \mathrm{kpa}$ stress and stress - strain curve of young and elderly groups were obtained, and regression analysis was used to develop the stress - strain relation expression, quantitatively compared the mechanical properties of the two groups, and discussed the relations of atherosclerosis and cerebral infarction from the biomechanical view, providing the 
biomechanical basis of the pathogenesis of atherosclerosis and cerebral infarction.

There was innovation in the experimental methods and data processing methods.

\section{Conflict of interest}

The authors declare that they have no conflict of interest

\section{ACKNOWLEDGEMENTS}

This work was supported by the Jilin Provincial Science and Technology Development Program (20110492). 


\section{REFERENCES}

1. Dong L, Underhill HR, Yu W, Ota H, Hatsukami TS, Gao TL et al. Geometric and compositional appearance of atheroma in an angiographically normal carotid artery in patients with atherosclerosis. AJNR Am J Neuroradiol 2010; 31: 311-6.

2. Yang WI, Shim CY, Cho IJ, Chang HJ, Choi D, Jang Y et al. Dyssynchronous systolic expansion of carotid artery in patients with marfan syndrome. J Am Soc Echocardiogr 2010; 23: 1310-6.

3. Au K, Singh MK, Bodukam V, Bae S, Maranian P, Ogawa R et al. Atherosclerosis in systemic sclerosis: a systematic review and meta-analysis. Arthritis Rheum 2011; 63: 2078-90.

4. Costanzo P, Cleland JG, Vassallo E, Perronr-Filardi P. Questioning the predictive role of carotid intima-media thickness. Future Cardiol 2011; 7: 651-6.

5. Makris GC, Lavida A, Griffin M, Geroulakos G, Nicolaides AN. Three-dimensional ultrasound imaging for the evaluation of carotid atherosclerosis. Atherosclerosis 2011; 219: 377-83.

6. Weber C, Noels H. Atherosclerosis: Current pathogenesis and therapeutic options. Nat Med 2011; 17: 1410-22.

7. Zhao X, Underhill HR, Zhao Q, Cai J, Li F, Oikawa M et al. Discriminating carotid atherosclerotic lesion severity by luminal stenosis and plaque burden: acomparisonutilizinghigh-resolution magnetic resonanceimaging at 3.0 Tesla. Stroke 2011; 42: 347-53.

8. Degnan AJ, Young VE, Gillard JH. Advances in noninvasive imaging for 
evaluating clinical risk and guiding therapy in carotid atherosclerosis. Expert Rev Cardiovasc Ther 2012; 10: 37-53.

9. Herder M, Johnsen SH, Amtzen KA, Mathiesen EB. Risk factors for progression of carotid intima-media thickness and total plaque area: a 13-year follow-up study. Stroke 2012; 43: 1818-23.

10. Roger VL, Go AS, Lloyd-Jones DM, Benjamin EJ, Berry JD, Borden WB et al. Heart disease and stroke statistics--2012 update: a report from the American Heart Association. Circulation 2012; 125: e2-e220

11. Spence JD. Carotid plaque measurement is superior to IMT Invited editorial comment on: carotid plaque, compared with carotid intima-media thickness, more accurately predicts coronary artery disease events: a meta-analysis-Yoichi Inaba, M.D., Jennifer A. Chen M.D., Steven R. Bergmann M.D., Ph.D. Atherosclerosis 2012; 220: 34-5.

12. Amin FM, Asghar MS, Ravneberg JW, de Koning PJ, Larsson HB, Olesen J et al. The effect of sumatriptan on cephalic arteries: A 3T MR-angiography study in healthy volunteers. Cephalalgia 2013; 33: 1009-16.

13. Homburg PJ, Rozie S, van Gils MJ, Jansen T, de Weert TT, Dippel DW et al. Atherosclerotic plaque ulcerationin the symptomatic internal carotid artery is associated withnonlacunar ischemic stroke. Stroke 2010; 41: 1151-6.

14. Simova I, Katova T, Denchev S. Diagnostic accuracy of flow-mediated dilatation and intima-media thickness for the presence of significant coronary artery disease. J Am Soc Hypertens 2009; 3: 388-94. 
15. Parmar JP, Rogers WJ, Mugler JP 3rd, Baskurt E, Altes TA, Nandalur KR et al. Magnetic resonanceimaging of carotid atherosclerotic plaque in clinically suspectedacute transient ischemic attack and acute ischemic stroke. Circulation 2010; 122: 2031-8.

16. Sadekova N, Vallerand D, Guevara E, Lesage F, Girouard H. Carotid calcification in mice: a new model to study the effects of arterial stiffness on the brain. J Am Heart Assoc 2013; 2: e000224.

17. Khorvash F, Rahimi M, Bagherian-Sararoudi R, Mousavi SA, Ebneshahidi A, Amirpour A et al. Evaluation of intima media thickness of carotid arteries in 40-60 years old persons with type d personality and its comparison with normal ones. Int J Prev Med 2013; 4: S250-5.

18. Von Hafe P, Lopes C, Maciel MJ, Barros H. [The clustering of cardiovascular risk factors in the urban population of Porto]. Acta Med Port 1998; 11: 1059-64.

19. Jackson ZS, Dajnowiec D, Gotlieb AI, Langille BL. Partial off-loading of longitudinal tension induces arterial tortuosity. Arterioscler Thromb Vasc Biol 2005; 25: 957-62.

20. Liu GY, Zhang Q, Jin Y, Gao ZL. Autogous nerve anastomosis versus humanamniotic membrane anastomosis a rheological comparison following simulated sciatic nerve injury. Neural Regen Res 2011; 6: 2424-8.

21. Liu GY, Zhang Q, Jin Y, Gao ZL. Stress and strain analysis on the anastomosis site sutured with either epineurial or perineurial sutures after simulation of sciatic nerve injur. Neural Regen Res 2012; 7: 2299-304. 
Quin et al

22. Peng CG, Zhang Q, Yang Q, Zhu QS. Strain and stress variations in the human amniotic membrane and fresh corpse autologous sciatic nerve anastomosis in a model of sciatic nerve injury. Neural Regen Res 2012; 7: 1779-85. 
Table: Longitudinal tensile results of carotid artery specimens of young and elderly groups.

\begin{tabular}{lll}
\hline Index & Young group & Elderly group \\
\hline Maximum stress (KPa) & $254.8 \pm 13.1$ & $227.2 \pm 7.3^{\mathrm{a}}$ \\
Maximum strain (\%) & $90.6 \pm 10.6$ & $67.7 \pm 5.0^{\mathrm{a}}$ \\
Elastic limit strain at $16.0 \mathrm{Kpa}$ & $39.5 \pm 2.8$ & $29.3 \pm 2.6^{\mathrm{a}}$ \\
Elastic limit strain at $13.3 \mathrm{Kpa}$ & $93.95 \pm 5.6$ & $98.5 \pm 1.8^{\mathrm{a}}$ \\
Elastic modulus at $16.0 \mathrm{Kpa}$ & $96.3 \pm 1.4$ & $102.5 \pm 2.3^{\mathrm{a}}$ \\
Elastic modulus at $22.5 \mathrm{Kpa}$ & $98.4 \pm 1.1$ & $106.1 \pm 1.5^{\mathrm{a}}$
\end{tabular}

Note: The data were expressed as $\mathrm{x} \pm \mathrm{s}$, the group comparison was performed using Sceffe France, ${ }^{\mathrm{a}} P<0.01$, vs young group. 


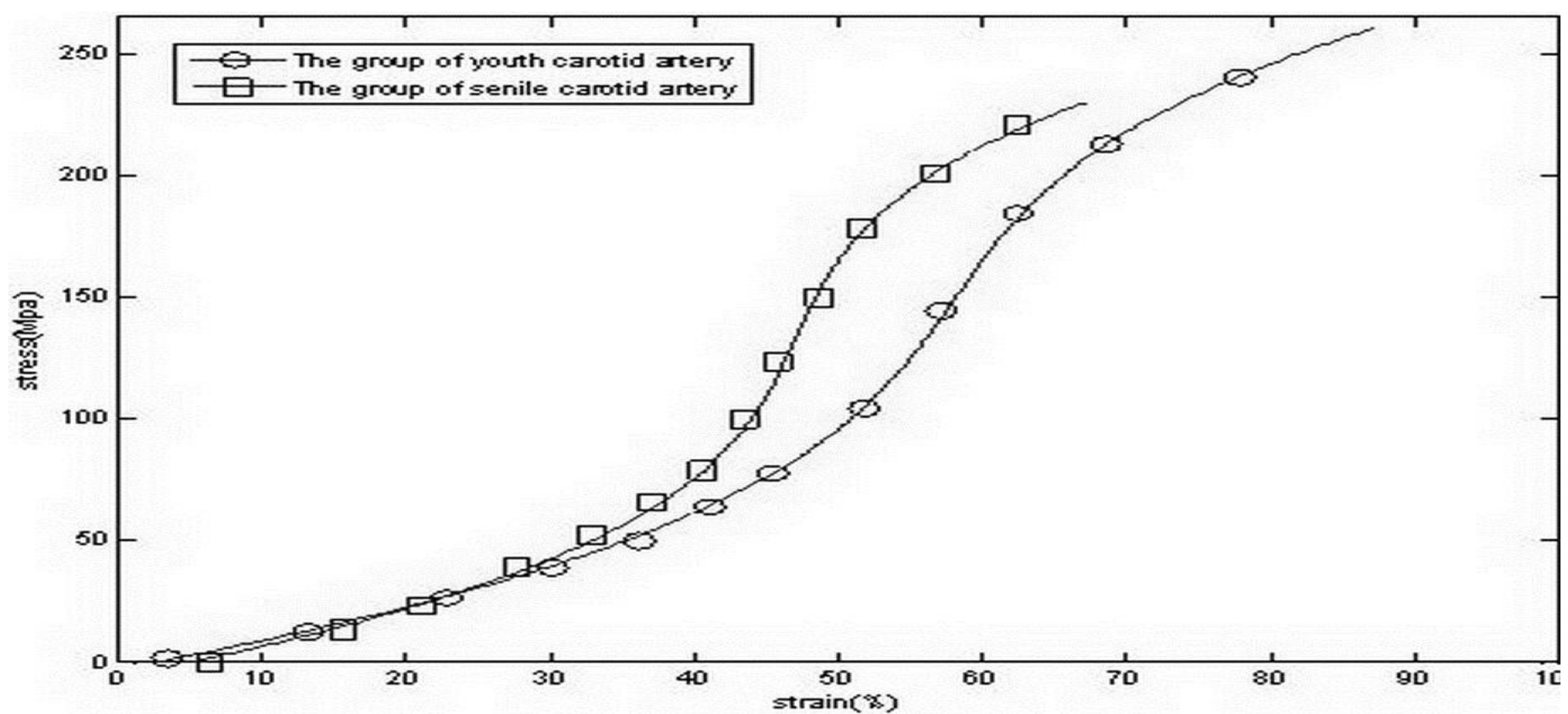

Fig.1: Stress-strain curve of longitudinal tensile in both young and elderly groups. 


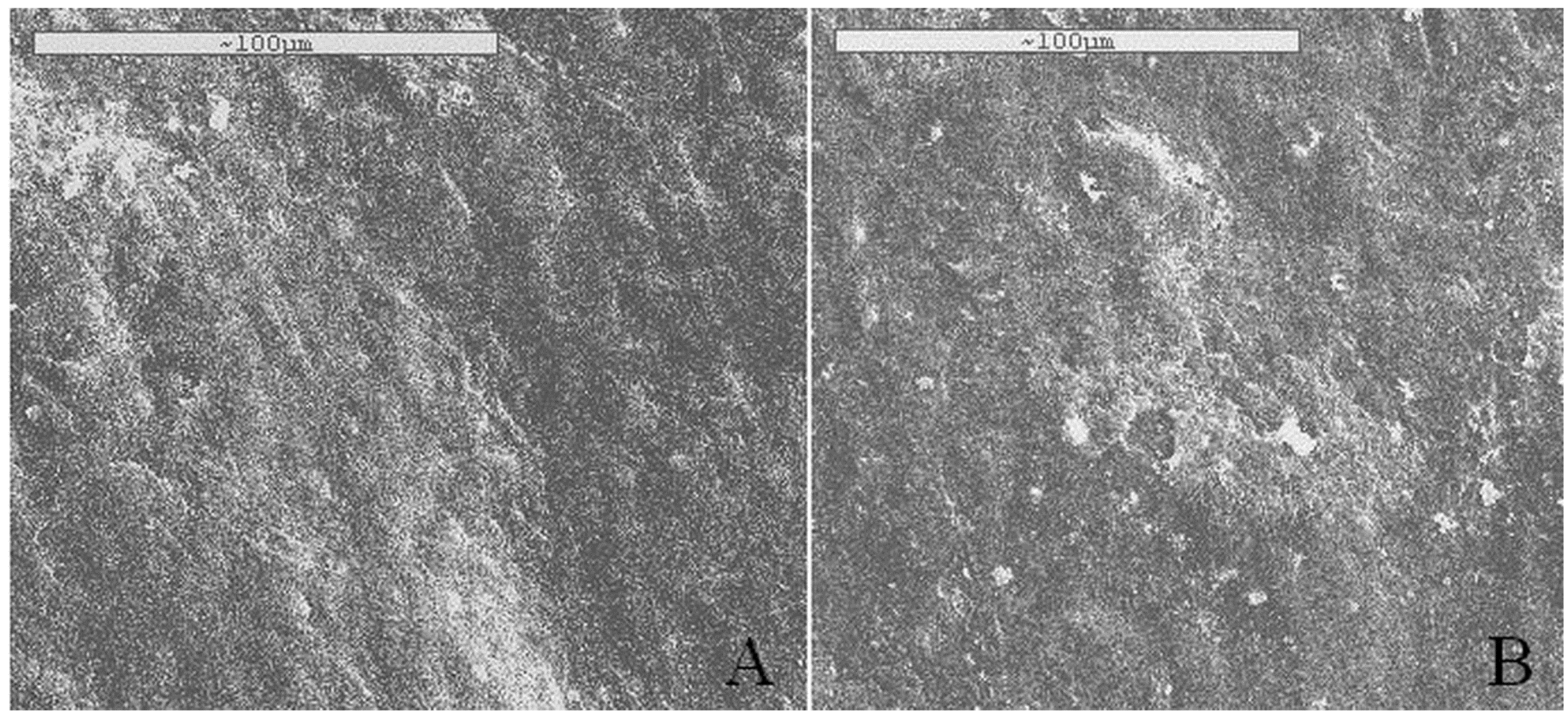

Fig.2: Longitudinal optical micrograph of young group. 


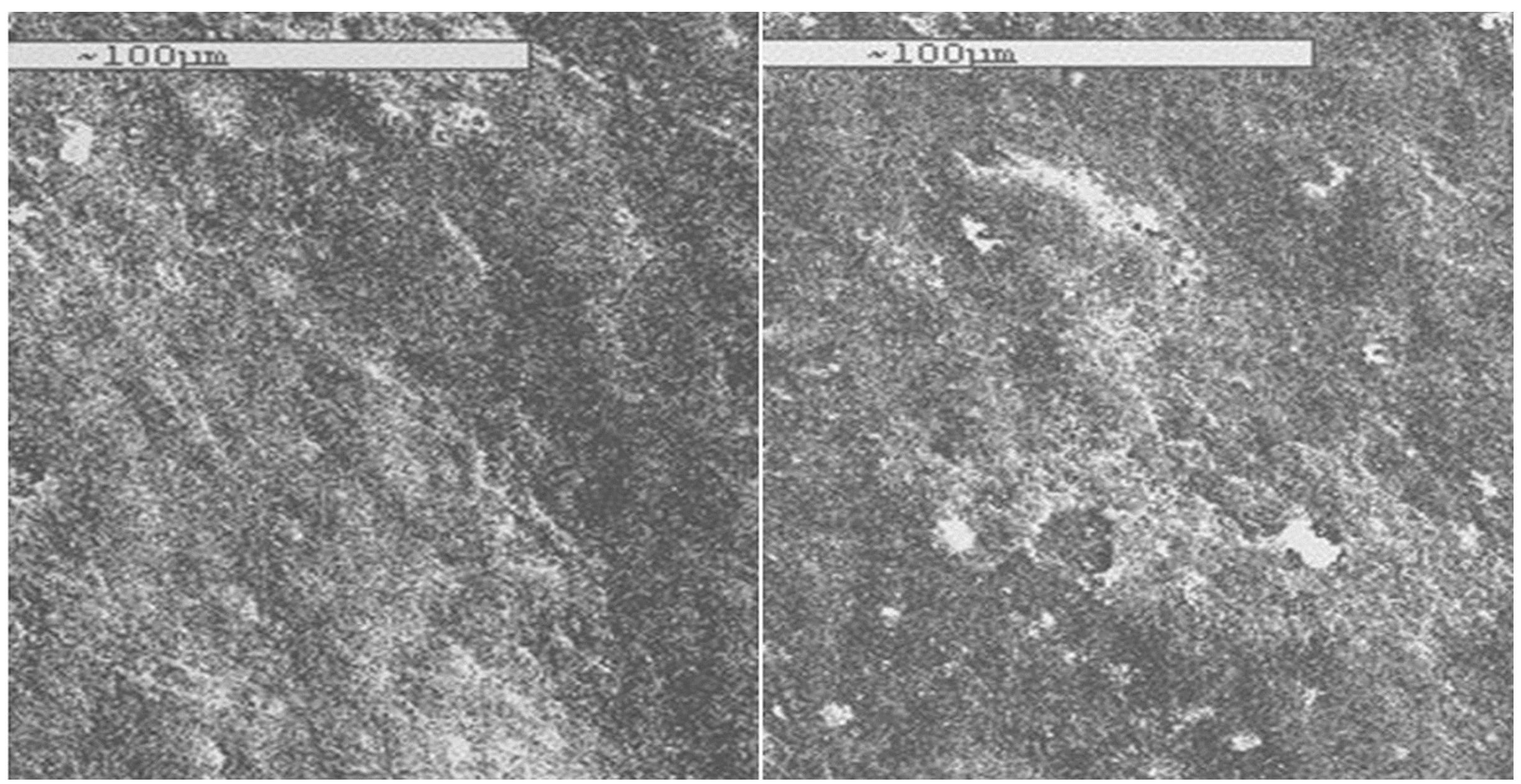

Fig.3: Longitudinal optical micrograph of elderly group. 